\title{
Iris metastasis as the first sign of small cell lung cancer: A case report
}

\author{
SHI-LIANG LIU, YU-HONG NIE, TAO HE, XI-XI YAN and YI-QIAO XING \\ Eye Center, Renmin Hospital of Wuhan University, Wuhan, Hubei 430060, P.R. China
}

Received August 5, 2015; Accepted September 16, 2016

DOI: $10.3892 / \mathrm{ol} .2017 .5648$

\begin{abstract}
The current study documents an unusual case of iris metastasis as the first sign of small cell lung cancer (SCLC) and reviews the relevant literature to increase understanding and awareness of this rare condition. A 59-year-old male chronic smoker presented with a painful and red right eye without any disturbance of vision. The patient also complained of associated chronic cough and anorexia. Upon examination, an exfoliative, pinkish-white lesion of the right iris was observed. A systemic work-up was performed, which incidentally detected metastatic SCLC in the lower lobe of the right lung and was confirmed by histopathological examination. A thorough evaluation demonstrated no organ metastases, except in the iris. Following the failure of conservative approaches, the eye had to be enucleated. The clinical features, treatment and prognosis of this condition are briefly reviewed and the results of contrast-enhanced ultrasound (CEUS) examination observed in this case are presented. Despite their rarity, iris lesions should be considered as possible manifestations of underlying malignancies. Imaging techniques, particularly CEUS, may aid the detection, diagnosis and monitoring of such lesions.
\end{abstract}

\section{Introduction}

Ocular metastasis is an increasingly recognized condition due to improved diagnostic accuracy and the use of whole-body imaging in patients with cancer. Choroidal metastasis is the most frequent type, whereas metastases in the iris, ciliary body, retina, vitreous and optic disc are all comparatively rare (1). Metastatic carcinoma of the iris may be one of the first indications of disseminated malignant disease, and its accurate differential diagnosis is important due to poor patient prognosis (2). Novel imaging methods, including ultrasonography

Correspondence to: Dr Yi-Qiao Xing, Eye Center, Renmin Hospital of Wuhan University, 238 Jiefang Road, Wuhan, Hubei 430060, P.R. China

E-mail: xyqdr07@sina.com

Key words: contrast-enhanced ultrasound, iris metastasis, secondary glaucoma, small cell lung cancer (color Doppler) and contrast-enhanced ultrasound (CEUS), have been developed not only to improve the early detection of malignant infiltrations, but also to improve the evaluation of tumor vascularity to diagnose an unknown primary. The present study describes a case of small cell lung cancer (SCLC), which initially presented as elevated intraocular pressure in the right eye with iris metastasis. The clinical, imaging and histological features are briefly described and a recently developed ultrasound technique, CEUS, was used to analyze the patient. The patient provided written informed consent.

\section{Case report}

A 59-year-old male was referred to the Eye Center, Renmin Hospital of Wuhan University (Wuhan, China) in April 2014, presenting with a 6-month history of worsening pain and redness in the right eye. The patient had been a chronic smoker for $\sim 40$ years and had developed an associated chronic cough. There was no history of significant weight loss; however, the patient did report suffering from anorexia. There was no other significant medical history.

The patient underwent an eye examination. Best-corrected visual acuity was 20/30 oculus dexter (OD) and 20/40 oculus sinister (OS). Intraocular pressure (IOP) was measured by a non-contact tonometer and was $37.2 \mathrm{mmHg}$ OD and $15.0 \mathrm{mmHg}$ OS. Slit lamp examination revealed circumciliary congestion, a transparent cornea, 2+ anterior chamber cells and a pinkish-white, vascularized iris mass at 10 o'clock to 1 o'clock meridians OD (Fig. 1). The swelling involved the upper region of the anterior chamber and reached up to the pupillary margin, subsequently distorting it. The patient reported that the swelling had rapidly enlarged over the past month. The pupillary diameter of the right eye was $2.5 \mathrm{~mm}$ and the pupillary light reflex was partly restricted. A gonioscopy was performed and neovascularization of the anterior chamber angle was reported OD. Fundoscopy of the right eye was attempted, however, it was unsuccessful due to pupillary occlusion and the presence of a cataract. Examination of the left eye did not identify any significant abnormalities. B-scan ultrasonography did not observe any space-occupying lesions at the posterior segment oculus uterque. Each eye exhibited a normal appearance and clear reflection of the posterior contour in B-scan ultrasonography.

The length, width and thickness of the iris tumor as measured by ultrasound biomicroscopy were 5.54, 3.72 and 

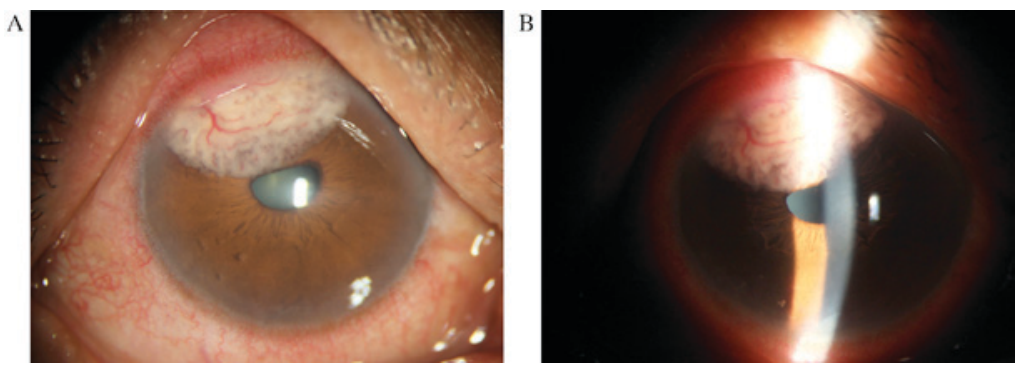

Figure 1. Iris metastasis from small cell lung cancer. (A) A vegetative, pink-white lesion with prominent vessels was observed on the surface of the right iris. (B) A slit lamp photograph of the iris metastasis.

$2.28 \mathrm{~mm}$, respectively. Angle closure due to tumor compression was also detected (Fig. 2). Orbital magnetic resonance imaging (MRI) demonstrated similar observations (Fig. 3), exhibiting equal $\mathrm{T} 1$ and $\mathrm{T} 2$ signals on the iris of the right eye, with no evident enhancement following contrast administration. CEUS was performed using the second-generation contrast agent SonoVue ${ }^{\circledR}$ (Bracco Imaging S.p.A., Milan, Italy) (Fig. 4) and a detailed evaluation of the vascularity was performed. Inhomogeneous enhancement in the first 18-24 s (rings), a hypoenhancement pattern and rapid washout in the early phase were noted in the anterior chamber of the right eyeball in the iris region. All findings were documented following each ophthalmic examination. Daily serial physical examinations were performed, during which a rapid enlargement of the mass was observed. After one week, the length, width and thickness of the iris tumor as measured by ultrasound biomicroscopy had increased to 5.81, 4.13 and $2.95 \mathrm{~mm}$, respectively.

Based on the afore mentioned findings, a diagnosis of primary tumor or metastasis to the iris was considered. Systemic physical examination was unremarkable. The lungs were clear on auscultation bilaterally, without marked wheezes, rales or rhonchi. However, a chest X-ray unexpectedly revealed bulky right hilum and right lower lobe segmental consolidation (Fig. 5). A computerized tomography (CT) scan identified a mass in the right lung measuring $6.0 \times 4.2 \mathrm{~cm}$ with hilar and mediastinal lymphadenopathy, indicative of malignancy (Fig. 6). A transbronchial biopsy was recommended and conducted based on the CT results. Histopathological examination, in addition to immunohistochemistry of the transbronchial biopsy (April 2014), suggested that the patient was suffering from SCLC. No other metastatic sites were detected following systematic evaluation, chest and abdominal $\mathrm{CT}$, head MRI and bone scintigraphy. The iris mass was therefore considered to be metastatic in nature.

Following serial examination, the iris swelling was observed to have rapidly enlarged. Despite 14 days of therapy with topical $1.0 \%$ carteolol hydrochloride (twice a day; China Otsuka Pharmaceutical Co., Ltd., Tianjin, China), 0.2\% brimonidine tartrate (twice a day; Allergan, Plc, Dublin, Ireland), oral methazolamide (100 mg/day; Hangzhou Aoyipollen Pharmaceutical Co., Ltd., Hangzhou, China) and 20\% mannitol intravenous injection (twice a day; Shandong Qidu Pharmaceutical Co., Ltd., Zibo, China) intravenous injection, IOP continued to be poorly controlled with progression of symptoms, including severe intractable pain. Refractory glaucoma aggravated corneal decompensation, which resulted in

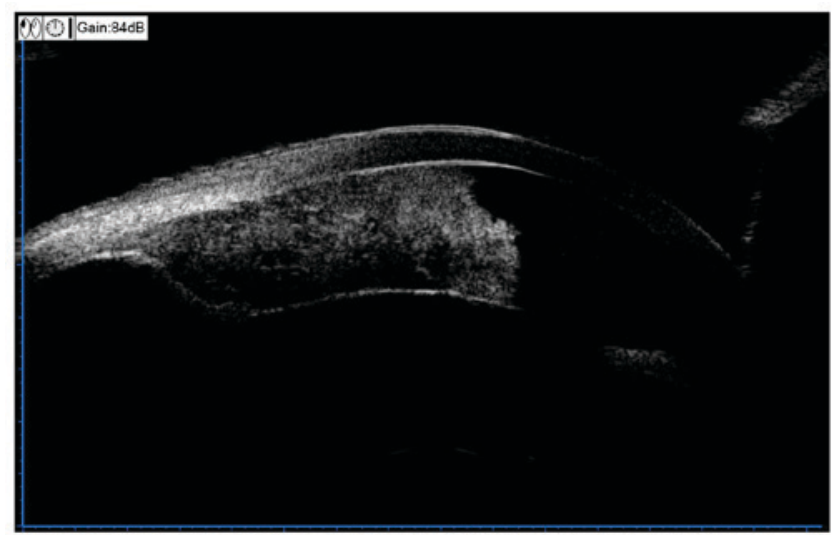

Figure 2. Ultrasound biomicroscopy of iris metastasis from small cell lung cancer, which revealed an irregular thickening of the inferior portion of the iris composed of medial internal reflectivity with invasion of the irido-corneal angle.

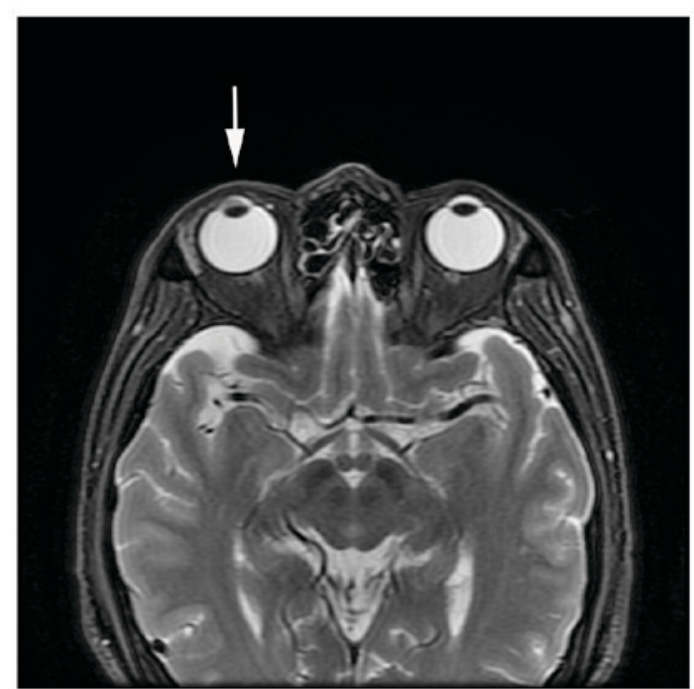

Figure 3. Orbital magnetic resonance imaging of iris metastasis from small cell lung cancer, indicating an equal $\mathrm{T} 1$ and equal $\mathrm{T} 2$ signal (arrow) on the iris of the right eye with no evident enhancement following contrast administration.

blurred vision over a 2-week period prior to SCLC diagnosis (during which no anti-cancer chemotherapy was administered). To prevent further deterioration of the eye, treatment with intravitreal bevacizumab (IVB) was recommended. However, due to financial constraints, the patient could afford 

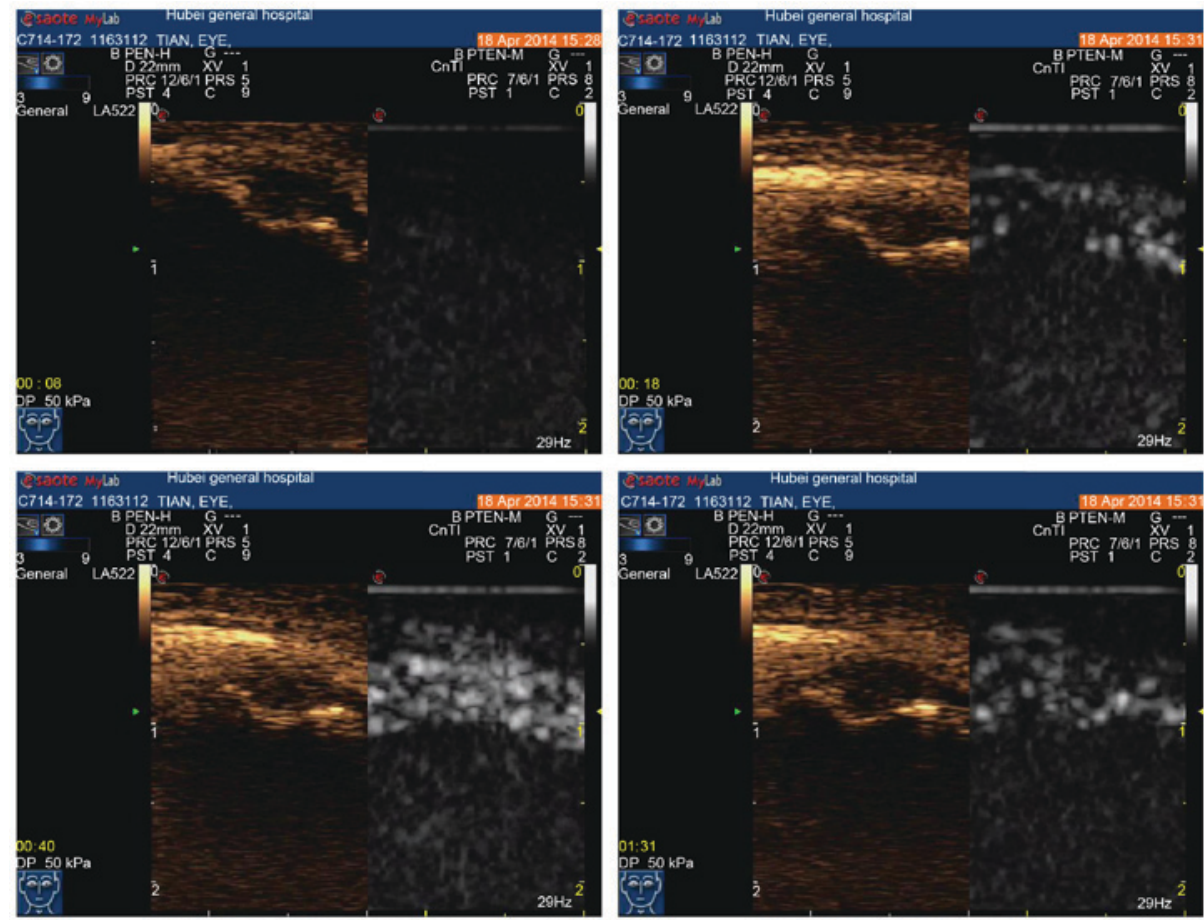

Figure 4. Contrast-enhanced ultrasound of the iris lesion. Inhomogeneous enhancement in the first 18-24 s (rings), a hypo-enhancement pattern and rapid washout in the early phase were noted in the anterior chamber of the right eyeball in the iris region.

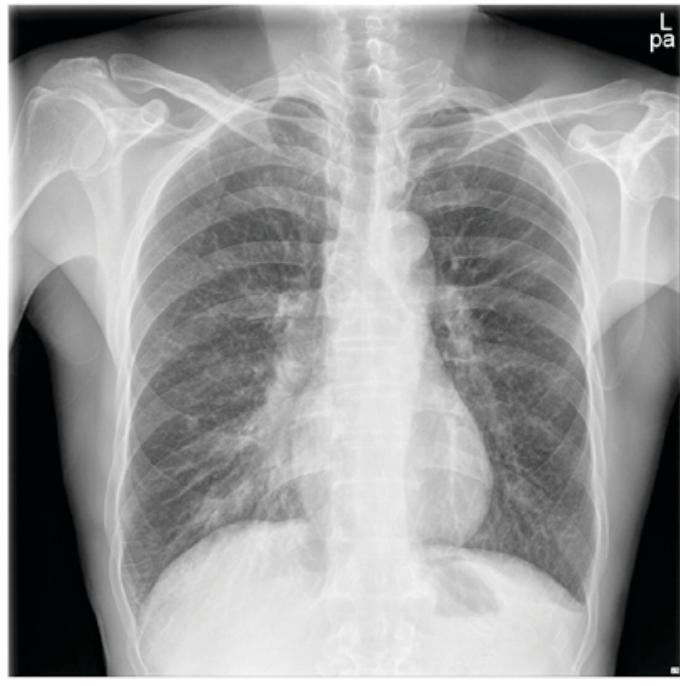

Figure 5. Chest X-ray revealed right hilar fullness and consolidation involving the paracardiac location of the right lower zone without silhouetting of the right heart border.

only conventional treatment. Hence, written informed consent was obtained for enucleation of the right eye.

Histopathological examination of the iris mass identified sheets and clusters of small cells with scanty cytoplasm and inconspicuous nucleoli, suggestive of small cell carcinoma (Fig. 7). The diagnosis of SCLC with iris metastasis was confirmed by immunohistochemical evaluation (Table I). All antibodies used in the immunohistochemical analysis were obtained from Fuzhou Maxim Biotech Co.,Ltd, Fuzhou, China. Tumor cells expressed cluster of differentiation 56, thyroid transcription factor-1, neuron-specific esterase and chromogranin;

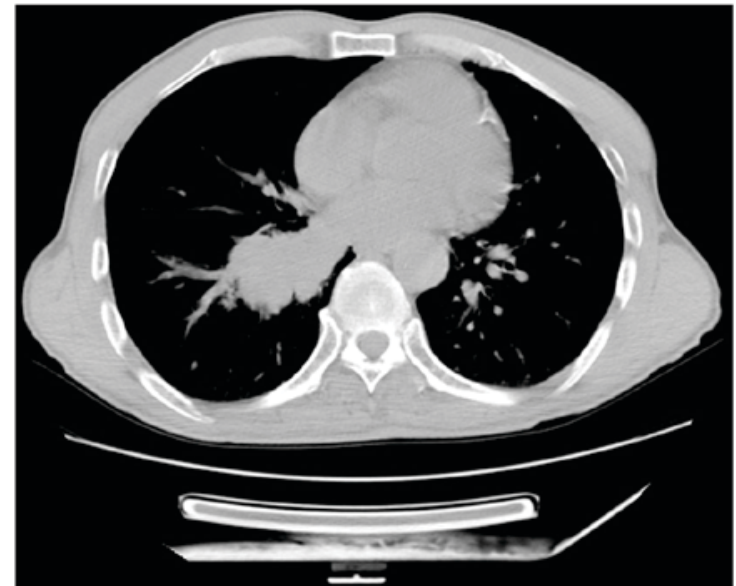

Figure 6. Computed tomographyof the chest showed a $6.0 \times 4.2 \mathrm{~cm}$ mass at the right hilus and mediastinal lymphadenopathy.

however, the cells did not express synaptophysin, cytokeratin (CK) 5/6, CK34ßE12 and p63 expression. A similar test was performed on the primary tumor in the lung, and the combined clinical, imaging and histological results confirmed the diagnosis of SCLC with iris metastasis.

Following enucleation, the patient was referred to an oncologist for further management. Combined chemotherapy of cisplatin $\left(80 \mathrm{mg} / \mathrm{m}^{2}\right.$, day 1) and etoposide $\left(100 \mathrm{mg} / \mathrm{m}^{2}\right.$, days 1, 2 and 3) (both Qilu Pharmaceutical Co., Ltd., Jinan, China) was administered over a 21-day cycle. Following one cycle of chemotherapy, the patient developed leukopenia (decreased white blood cell count, $1.63 \times 10^{9}$ cells/1; normal range, $3.5-9.5 \times 10^{9}$ cells $/ 1$ ), increasing the risk of infection, therefore further treatment was refused. No radiation to the 
Table I. Immunohistochemical profile of the iris lesion.

\begin{tabular}{ll}
\hline Marker & Result \\
\hline CD56 & Positive \\
TTF-1 & Positive \\
NSE & Positive \\
CgA & Positive \\
Ki67 & Positive \\
Syn & Negative \\
CK5/6 & Negative \\
CK34ßE12 & Negative \\
p63 & Negative \\
\hline
\end{tabular}

$\mathrm{CD}$, cluster of differentiation; TTF-1, thyroid transcription factor-1; NSE, neuron-specific esterase; CgA, chromogranin; Syn, synaptophysin; CK, cytokeratin.

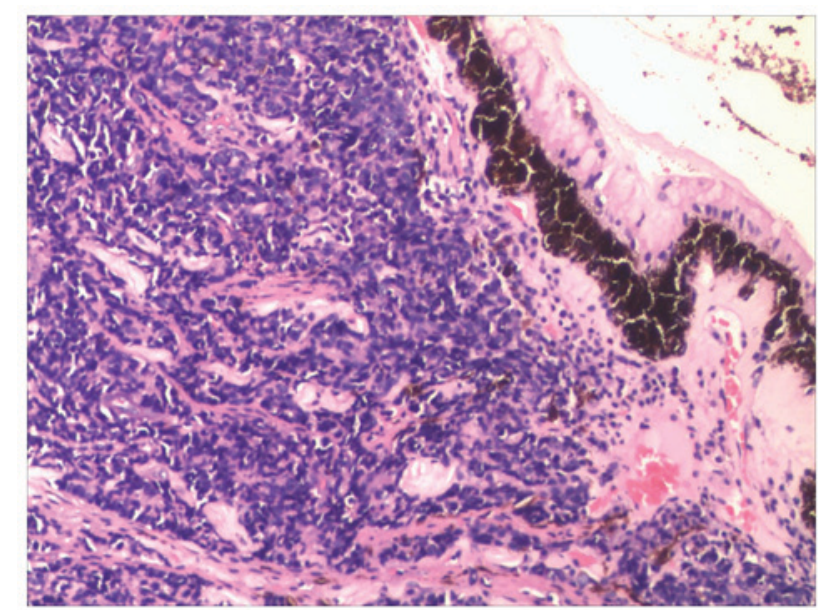

Figure 7. Hematoxylin and eosin staining of the iris lesion identified cells with round or elongated nuclei arranged in clusters with several areas of necrosis. Inconspicuous nucleoli, focal areas of clumped chromatin and several typical and atypical mitotic figures were observed. Magnification, x200.

lung, orbit or the brain was administered. One year after the initial diagnosis, the patient is asymptomatic and is followed up regularly every 3 months. The opposite eye is currently asymptomatic and normal on examination, and no changes of atrophy are visible.

\section{Discussion}

Metastatic carcinoma is the most frequent intraocular malignancy, and most commonly originates from breast and lung carcinoma (3). Previous studies have demonstrated that lung cancer of either non-small cell or small cell type may metastasize to the eye (4) and present without any preceding manifestations of primary cancer (5-8), as observed in the current study. SCLC constitutes $\sim 20 \%$ of all lung cancer cases and is characterized by worsening patient prognosis due to its rapid growth and early metastasis (9). Iris involvement accounts for only $7.8-10 \%$ of all uveal metastases $(3,8,10,11)$, and they generally coexist with central nervous system metastases in $\sim 44 \%$ of cases (10). A few studies regarding distant metastases occurring exclusively in the iris have been published $(12,13)$; however, this is rare and the best approach to its clinical recognition and treatment remains to be elucidated. Diagnosing iris metastasis therefore requires a high index of suspicion along with careful review of the clinical history and complete ocular examination.

Pain and blurred vision are the most common symptoms for patients with iris metastasis (14). Tumors are likely to be unilateral and may be associated with a variety of other symptoms, including glaucoma, abnormal vascularization, hyphema, ectropion, pigment dispersion, pseudohypopyon, iridocyclitis and pupillary distortion (15). A careful evaluation of any underlying malignancy is warranted if a patient exhibits unilateral anterior uveitis or intractable glaucoma (2).

The quality of life and overall survival of patients with isolated iris metastasis may be improved by prompt diagnosis and treatment. High-quality photographs are used to document the surface characteristics of iris metastasis. Tumor thickness, size and boundaries are typically determined by ultrasound biomicroscopy or anterior segment optical coherence. High resolution CT and MRI scans serve as non-invasive imaging methods with the potential to distinguish malignant lesions from benign masses, including foreign bodies, massive hemorrhage and cystic lesions (16). However, iris metastasis may not always be easily distinguished from an achromic melanoma, particularly when the primary cancer is unknown (7). In such cases, a fine-needle aspiration biopsy may aid accurate diagnosis.

A number of studies have demonstrated the utility of conventional ultrasonography (B-mode and color Doppler) in the initial work-up of intraocular metastases $(7,17)$. Differentiating malignant from benign iris masses relies primarily on topographic location, analysis of pattern and degree of vascularity. CEUS provides a precise description of the vascularization of any lesion, and its importance has been proven in the detection of adrenal, oral and lymph node cancer $(18,19)$. Several preliminary studies on the role of CEUS in the characterization of peripheral lung masses have been performed (20-22). The application of CEUS facilitates the visualization of vascularization and perfusion, and may aid more accurate differentiation between characteristic features of benign and malignant changes. However, the role of CEUS in detecting iris masses has not been previously evaluated.

In the current study, inhomogeneous enhancement in the first 18-24 s (rings), a hypoenhancement pattern and rapid washout were all noted in the early phase of the iris tumor development. The utility of CEUS is much higher when clinical information is included in image interpretation, but such data are challenging to summarize as a complex clinical picture is often encountered (18). The present case highlights that delayed time to enhancement, a hypoenhancement pattern on CEUS and rapid washout in the early phase may all suggest an underlying malignancy as the primary diagnosis. Such an imaging modality has the potential to reduce the requirement for biopsy and may aid the screening and follow-up of incidentally diagnosed iris masses.

Following the diagnosis of iris metastasis, treatment decisions typically depend on the tumor size, location, secondary 
complications, patterns of growth and local extension (16). Patients should be promptly referred to a general oncologist and onco-ophthalmologist for further treatment and systemic examination (23). Generally, treatment of anterior segment tumors usually consists of ocular conservation and treatment options for iris metastasis typically include surgical excision, plaque radiotherapy, external beam radiotherapy or systemic chemotherapy (2). Ocular outcome in patients with iris metastasis is largely favorable in $95 \%$ of cases (2); however, overall prognosis is determined by systemic neoplastic disease. Therefore, clinicians must balance optimism with a more realistic outlook of a shortened patient lifespan. Enucleation should only be performed if palliative care is ineffective in controlling secondary glaucoma.

Glaucoma secondary to intraocular malignancy is challenging to diagnose, and often refractory to treatment. Consistent with previous reports, secondary glaucoma may have developed in the present case through a variety of mechanisms, including infiltration and invasion of the trabecular meshwork, secondary angle closure, neovascularization and tumor-induced uveitis. Additional cytological examination of aqueous humor is suggested to confirm the cause.

The management of eye cancer-associated glaucoma varies and is dependent on the primary cancer, ocular symptoms, overall cancer presentation and estimated life expectancy. Treatment options typically include the administration of standard medication, laser, incisional and radiotherapeutic approaches (24). Treatment goals consist of protecting or improving patient vision in order to avoid enucleation and maintain a good quality of life.

IVB, an intravenously administered monoclonal antibody against vascular endothelial growth factor, has been approved for systemic use in various metastatic malignancies (25). There commended dose ranges from $1.25-4 \mathrm{mg}$, and it has proven to be a safe, effective and relatively easy local treatment for uveal metastasis (17,26-31). However, a number of patients with choroidal metastases originating from a variety of primaries, including the lung, colon and breast, experience progressive metastasis despite undergoing IVB treatment (32). A previous case report documented the occurrence of intravitreal hemorrhage 20 months after the first IVB injection (33). The effectiveness, appropriate dosing and long-term outcomes of IVB should therefore be further evaluated.

In conclusion, the present study described a rare case of iris metastasis presenting as the initial manifestation of SCLC. A high index of suspicion, coupled with a detailed clinical history and findings collected from imaging examination are necessary in cases such as these to diagnose the unknown primary. The specific enhancement pattern of CEUS may aid the characterization, treatment choice and therapeutic monitoring of iris metastasis. Early diagnosis and appropriate treatment are required to improve the outcome of such patients.

\section{Acknowledgements}

The present study was funded by the National Natural Science Foundation of China (grant no. 81271025) and the National Health and Family Planning Commission of China (grant no. 201302015).

\section{References}

1. De Potter P: Ocular manifestations of cancer. Curr Opin Ophthalmol 9: 100-104, 1998.

2. Shields CL, Kaliki S, Crabtree GS, Peshtani A, Morton S, Anand RA, Coco G and Shields JA: Iris metastasis from systemic cancer in 104 patients: The 2014 Jerry A. Shields Lecture. Cornea 34: 42-48, 2015

3. Shields CL, Shields JA, Gross NE, Schwartz GP and Lally SE: Survey of 520 eyes with uveal metastases. Ophthalmology 104: 1265-1276, 1997.

4. Lampaki S, Kioumis I, Pitsiou G, Lazaridis G, Syrigos K, Trakada G, Kakolyris S, Zarogoulidis K, Mpoukovinas I, Rapti A and Zarogoulidis P: Lung cancer and eye metastases. Med Hypothesis Discov Innov Ophthalmol 3: 40-44, 2014.

5. Su HT, Chen YM and Perng RP: Symptomatic ocular metastases in lung cancer. Respirology 13: 303-305, 2008.

6. Kreusel KM,Bechrakis NE, Wiegel T, Krause L and FoersterMH: Incidence and clinical characteristics of symptomatic choroidal metastasis from lung cancer. Acta Ophthalmol 86: 515-519, 2008.

7. Meziani L, Cassoux N, Le Rouic LL, Gabriel CL, Dendale R, Sastre X, Catherine D, Livartowski A, Plancher C, Asselain B and Desjardins L: Uveal metastasis revealing lung cancer. J Fr Ophtalmol 35: 420-425, 2012.

8. Shah SU, Mashayekhi A, Shields CL, Walia HS, Hubbard GB III, Zhang J and Shields JA: Uveal metastasis from lung cancer: Clinical features, treatment, and outcome in 194 patients. Ophthalmology 121: 352-357, 2014.

9. Hoffman PC, Mauer AM and Vokes EE: Lung cancer. Lancet 355: 479-485, 2000.

10. Shields JA, Shields CL, Kiratli H and de Potter P: Metastatic tumors to the iris in 40 patients. Am J Ophthalmol 119: 422-430, 1995.

11. Soysal HG: Metastatic tumors of the uvea in 38 eyes. Can J Ophthalmol 42: 832-835, 2007.

12. Jurecka T, Skorkovská S, Coupková H and Postránecká V: Iris metastasis as the first sign of small-cell lung carcinoma with metastatic involvement of the mediastinum. Klin Onkol 22: 179-182, 2009 (In Czech).

13. Straub M, Muselier A, Bron A and Creuzot-Garcher C: An iris metastasis revealed a bronchopulmonary cancer. J Fr Ophtalmol 37: 125-129, 2014 (In French).

14. Ferry AP and Font RL: Carcinoma metastatic to the eye and orbit II. A clinicopathological study of 26 patients with carcinoma metastatic to the anterior segment of the eye. Arch Ophthalmol 93: 472-482, 1975 .

15. Kanthan GL, Jayamohan J, Yip D and Conway RM: Management of metastatic carcinoma of the uveal tract: An evidence-based analysis. Clin Experiment Ophthalmol 35: 553-565, 2007.

16. Marigo FA and Finger PT: Anterior segment tumors: Current concepts and innovations. Surv Ophthalmol 48: 569-593, 2003.

17. Augustine H, Munro M, Adatia F, Webster M and Fielden M: Treatment of ocular metastasis with anti-VEGF: A literature review and case report. Can J Ophthalmol 49: 458-463, 2014.

18. Dietrich CF, Ignee A, Barreiros AP, Schreiber-Dietrich D, Sienz M, Bojunga J and Braden B: Contrast-enhanced ultrasound for imaging of adrenal masses. Ultraschall Med 31: 163-168, 2010.

19. Cui XW, Hocke M, Jenssen C, Ignee A, Klein S Schreiber-Dietrich D and Dietrich CF: Conventional ultrasound for lymph node evaluation, update 2013. Z Gastroenterol 52: 212-221, 2014.

20. Sperandeo M, Sperandeo G, Varriale A, Filabozzi P, Decuzzi M, Dimitri L and Vendemiale G: Contrast-enhanced ultrasound (CEUS) for the study of peripheral lung lesions: A preliminary study. Ultrasound Med Biol 32: 1467-1472, 2006.

21. Caremani M, Benci A, Lapini L, Tacconi D, Caremani A, Ciccotosto C and Magnolfi AL: Contrast enhanced ultrasonography (CEUS) in peripheral lung lesions: A study of 60 cases. J Ultrasound 11: 89-96, 2008.

22. Sartori S, Postorivo S, Vece FD, Ermili F, Tassinari D and Tombesi P: Contrast-enhanced ultrasonography in peripheral lung consolidations: What's its actual role? World J Radiol 5: 372-380, 2013.

23. Miller J: Metastatic ocular tumors of the anterior segment. Optometry 79: 189-192, 2008.

24. Radcliffe NM and Finger PT: Eye cancer related glaucoma: Current concepts. Surv Ophthalmol 54: 47-73, 2009. 
25. Horn L, Dahlberg SE, Sandler AB, Dowlati A, Moore DF, Murren JR and Schiller JH: Phase II study of cisplatin plus etoposide and bevacizumab for previously untreated, extensive-stage small-cell lung cancer: Eastern Cooperative Oncology Group Study E3501. J Clin Oncol 27: 6006-6011, 2009

26. Nakashima C, Keino H, Watanabe T, Taki W and Okada AA Intravitreal bevacizumab for iris metastasis of small-cell lung carcinoma with neovascular glaucoma. Jpn J Ophthalmol 55 80-81, 2011.

27. Schell JC, Koenig SB, Bastin K and Wirostko WJ: Intracameral bevacizumab administered for non-small cell lung cancer metastasis to iris. Clin Pract 1: e39, 2011.

28. Yokouchi H, Kitahashi M, Oshitari T and Yamamoto S: Intravitreal bevacizumab for iris tumor metastasized from large cell neuroendocrine carcinoma of lung. Graefes Arch Clin Exp Ophthalmol 251: 2243-2245, 2013.

29. Zhou Q, Liang J and Lu H: Intravitreal bevacizumab for ocular metastasis of multiple myeloma. Optom Vis Sci 90: e236-e240, $1028,2013$.
30. Lin CJ, Li KH, Hwang JF and Chen SN: The effect of intravitreal bevacizumab treatment on choroidal metastasis of colon adenocarcinoma-case report. Eye (Lond) 24: 1102-1103, 2010.

31. Zako M, Takeyama M, Naito E, Mizumoto K and Iwaki M: Choroidal and optic disc metastases from breast cancer and their response to combination pharmacotherapy with tamoxifen, cyclophosphamide hydrate, letrozole, and bevacizumab. J Ocul Pharmacol Ther 28: 89-93, 2012.

32. Maudgil A, Sears KS, Rundle PA, Rennie IG and Salvi SM: Failure of intravitreal bevacizumab in the treatment of choroidal metastasis. Eye (Lond) 29: 707-711, 2015.

33. Fabrini MG, Genovesi-Ebert F, Perrone F, De Liguoro M, Giovannetti C, Bogazzi F, Rizzo S, Martino E and Cionini L: A multimodal approach to the treatment of bilateral choroidal metastases from thyroid carcinoma. Rare Tumors 1: e4, 2009. 